\title{
Mosaic structure and reflectivity of an as grown copper single crystal using neutron diffraction
}

\author{
MALA N RAO and A M SHAIKH* \\ Solid State Physics Division, Bhabha Atomic Research Centre, Bombay 400085, India \\ MS received 25 April 1996
}

\begin{abstract}
A single crystal of copper of size $80 \mathrm{~mm}$ dia. and $50 \mathrm{~mm}$ length was grown by the Bridgman method. Neutron diffraction investigations were carried out to determine the mosaic spread and reflectivity of this as grown crystal. The crystal was found to be of good quality having structure free and symmetric rocking curves with mosaic spread of 14 min.arc and reflectivity of $\sim 50 \%$.
\end{abstract}

Keywords. Crystal growth; neutron diffraction: copper: mosaic structure.

\section{Introduction}

Large metallic single crystals are often used as monochromators and analysers in neutron scattering experiments and it is desirable that these crystals have large mosaic spread and high reflectivity. It is therefore essential to know the diffraction properties of the crystals, prior to their use as monochromators.

It has been observed that the mosaic structure of relatively large crystals is often inhomogeneous. If such a crystal is used as a monochromator, these inhomogeneities result in an irregular intensity distribution over the cross-section of the neutron beam incident on the sample. Therefore, ideally a neutron monochromator should have homogeneous mosaic spread all over the volume and the mean orientation of the reflecting plane should be the same all over the crystal. The most commonly used monochromator/analyser crystals are beryllium, copper, germanium, silicon and Heusler alloy $\mathrm{Cu}_{2} \mathrm{MnAl}$.

As part of our programme on fabrication of monochromators and analysers we have grown a large copper crystal using Bridgman technique and investigated its mosaic structure and reflectivity characteristics using neutron diffraction. These investigations are reported in this paper.

\section{Theory}

Mosaicity in a single crystal arises because it is composed of mosaic blocks, each individually perfect, but oriented in a Gaussian distribution of the type

$$
W(\Delta)=\frac{1}{\eta \sqrt{2 \pi}} \exp \left\{-\frac{\Delta^{2}}{2 \eta^{2}}\right\} .
$$

where $\eta$ is the standard deviation of the distribution function and $\Delta$ the rocking angle of the crystal. The full width at half maximum of $W(\Delta)$ is called mosaic spread $\beta$ and is

\footnotetext{
*To whom all correspondence should be addressed.
} 
related to $\eta$ through

$$
\beta=2 \sqrt{(2 \ln 2)} \eta \text {. }
$$

In practice the mosaic spread of the crystal can be estimated from FWHM of its rocking curve, which is the intensity of the reflected beam plotted as a function of angle of orientation of the specimen around the Bragg angle ( $w$-scan). The theory of dependence of rocking curve width on Bragg angle has been derived by Caglioti and Ricci (1962) and is expressed by the relation

$$
\beta_{2}^{2}=B_{1 / 2}^{2}-\frac{A \alpha_{2}^{2} \alpha_{3}^{2}-2 B a \alpha_{2}^{2} \alpha_{3}^{2}+a^{2}\left[C \alpha_{3}^{2}+\alpha_{1}^{2} \alpha_{2}^{2} \beta_{1}^{2}\right]}{D \alpha_{3}^{2}+A \alpha_{2}^{2}-4 B a \alpha_{2}^{2}+4 C a^{2}},
$$

where $A=\alpha_{1}^{2}+4 \beta_{1}^{2}, B=\alpha_{1}^{2}+2 \beta_{1}^{2}, C=\alpha_{1}^{2} \alpha_{2}^{2}+\alpha_{1}^{2} \beta_{1}^{2}+\alpha_{2}^{2} \beta_{1}^{2}$ and $D=A+\alpha_{2}^{2}$, and $\beta_{1}$ : mosaic spread of the monochromator crystal, $\beta_{2}$ : mosaic spread of the sample crystal, $B_{1 / 2}$ : FWHM of the sample rocking curve, $\alpha_{i}$ : horizontal angular divergence of the $i$ th collimator, $i=1,2,3$ and is equal to the ratio of width to length of the Soller collimator. $a$ : Dispersion parameter $=\tan \theta / \tan \theta_{\mathrm{m}}$, where $\theta$ and $\theta_{\mathrm{m}}$ are the Bragg angles for the single crystal and the monochromator respectively.

When $\theta=\theta_{\mathrm{m}}$, the FWHM of the peak is independent of $\alpha_{i}$ 's and is solely determined by the mosaic spreads of the monochromator and sample crystal (Arndt and Willis 1966). In this case,

$$
B_{1 / 2}=2 \sqrt{\ln 2\left(\beta_{1}^{2}+\beta_{2}^{2}\right)} \text {. }
$$

The refiectivity of an ideal mosaic crystal can be calculated from the diffraction theory (Bacon and Lowde 1948) with the assumption that each mosaic block is so small that it does not significantly attenuate the primary beam by diffraction i.e. there is no primary extinction and secondly, that no interference takes place between different blocks because similarly oriented blocks are widely separated. The refiectivity $r(\Delta)$ as a function of rocking angle $\Delta$ for the symmetric case (Bragg case) is (Dorner 1971)

$$
r(\Delta)=\frac{1}{2}\left[1-\exp \left\{-2 Q W(\Delta) \frac{t}{\cos \theta}\right\}\right],
$$

and can be determined experimentally using the expression

$$
r(\Delta)=\frac{I_{\mathrm{d}}(\Delta)}{I_{0} \exp \{-\mu t / \cos \theta)},
$$

where $I_{0}$ and $I(\Delta)$ are the incident and diffracted intensities, $\mu$ the total absorption factor, $t$ the thickness of the crystal and $\theta$ the Bragg angle. $Q$ is the crystallographic quantity:

$$
Q=\frac{N^{2} \lambda^{3} F^{2}}{\sin 2 \theta}
$$

where $N$ is the number of unit cells per unit volume, $\lambda$ the wavelength of the incident neutrons, and $F$ is the structure factor of the reflection being considered.

The transmitted intensity in the forward direction is given by

$$
\frac{I_{\mathrm{f}}(\Delta)}{I_{0}}=\exp \left\{-\frac{\mu t}{\cos \theta}\right\}(1-r(\Delta)) .
$$




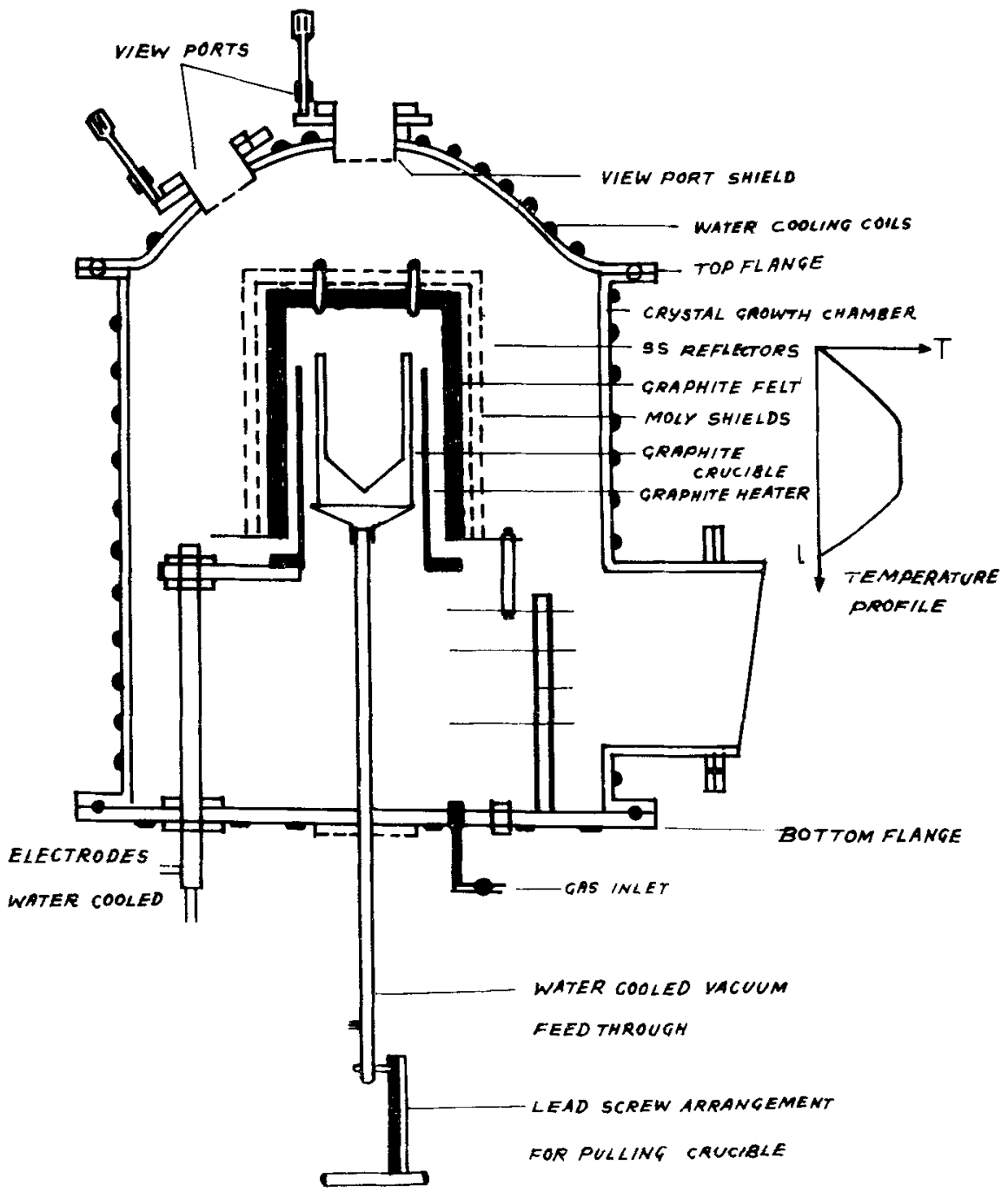

Figure 1. Schematic diagram of the Bridgman type crystal growth apparatus.

\section{Experimental}

A single crystal of copper was grown using Bridgman technique having stationary furnace and moving crucible. The experimental arrangement used is shown in figure 1. A vertical tubular furnace with graphite heating element (ID $=100 \mathrm{~mm}$ and length $150 \mathrm{~mm}$ ) was used. The temperature measured as a function of the radial distance was a convex isotherm with an uniform temperature zone of $50 \mathrm{~mm}$ width at the centre of the furnace with an uncertainty of $\pm 7 \cdot 5^{\circ} \mathrm{C}$ at highest operating temperature of $2000^{\circ} \mathrm{C}$. The temperature of the furnace was controlled through a programmed PID controller using a W-Re thermocouple and could be kept within $\pm 2{ }^{\circ} \mathrm{C}$ at $1500^{\circ} \mathrm{C}$.

An initial charge of OFHC copper ingot was kept in a graphite crucible having an inside diameter of $80 \mathrm{~mm}$ and a length of $150 \mathrm{~mm}$. The bottom of the crucible was 


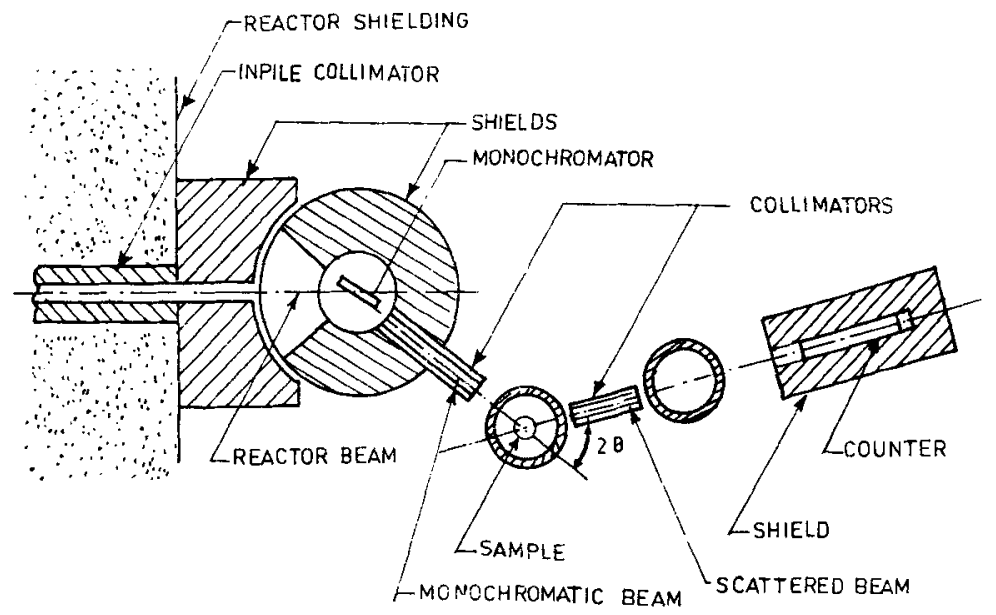

Figure 2. Schematic diagram of the neutron spectrometer used.

Table 1. Details of neutron spectrometer used.

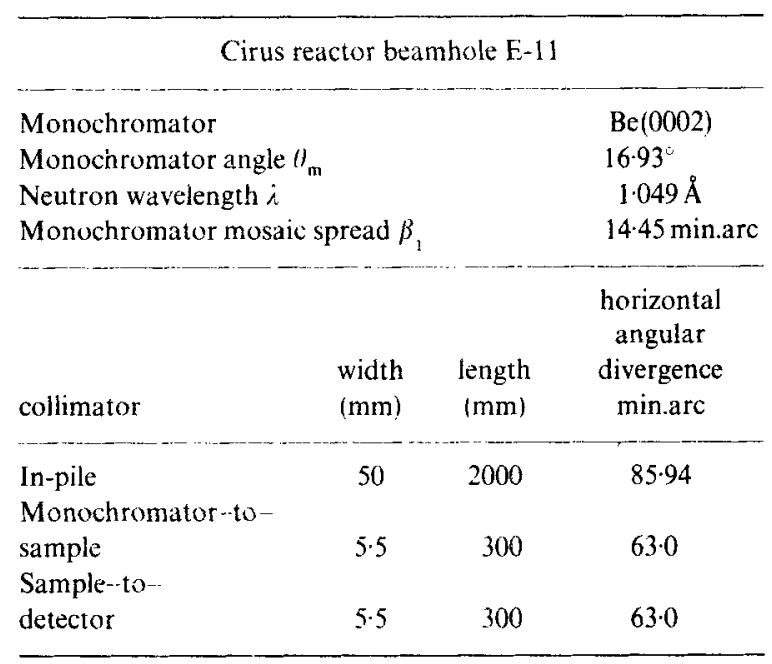

shaped to a conical tip with a taper angle of $90^{\circ}$. The crucible was kept on the pedestal inside the tubular furnace such that the copper ingot lay in the uniform zone of the furnace.

The vessel where $\mathrm{Cu}$ crystal was grown was evacuated for several hours and the resulting pressure was $10^{-5}$ Torr. The furnace was heated up from room temperature to $1150^{\circ} \mathrm{C}$, well beyond the melting temperature of copper $\left(1083^{\circ} \mathrm{C}\right)$, at a rate of $3^{\circ} / \mathrm{min}$. After holding the melt at this temperature for about an hour, the crucible was lowered at a rate of $5 \mathrm{~mm} / \mathrm{h}$, such that the molten mass passed through the temperature gradient of the furnace. The furnace was then cooled to room temperature at a rate of $2^{\circ} / \mathrm{min}$. The grown crystal was removed easily from the crucible. No reaction between the crystal and graphite crucible was observed during the growth process. The crystal, bright and deep red, was $80 \mathrm{~mm}$ in diameter and $50 \mathrm{~mm}$ in length. 


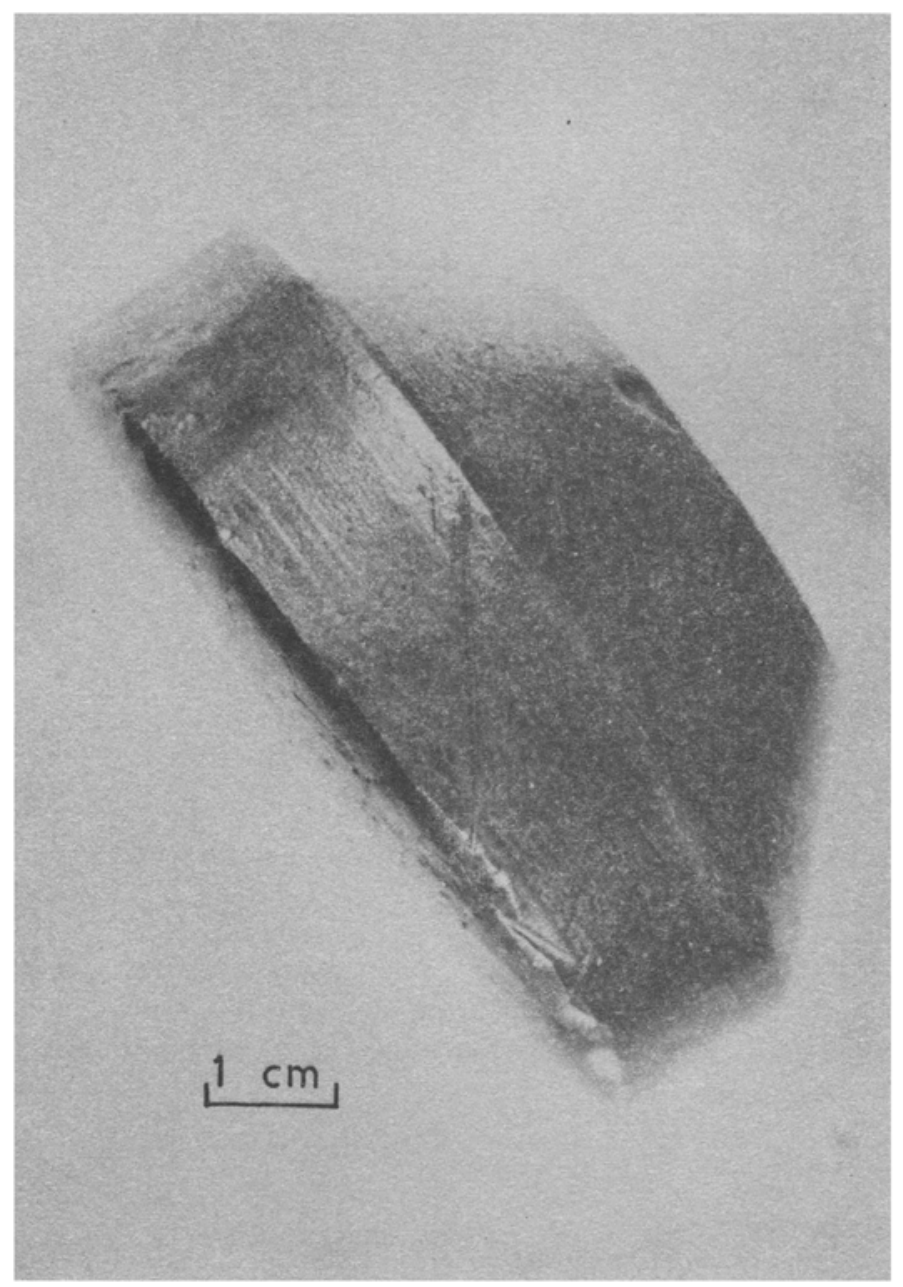

Figure 3. Photograph of the copper single crystal.

The characterization of the as grown crystal was carried out on a neutron spectrometer at Cirus reactor using a Be monochromated beam of neutrons of wavelength $1.049 \AA$ (figure 2 ). Table 1 gives the details of the spectrometer used. A small part of the crystal ( $\sim 15 \mathrm{~mm}$ in length) at the conical tip was sliced off before mounting it on the goniometer head. This was done to remove crystallites of different orientations that might have nucleated during the initial stages of crystal growth. Figure 3 shows a photograph of the crystal used for characterization.

The crystal was mounted with its growth direction parallel to the neutron beam direction. It was carefully centred and aligned and various reflections were scanned. This initial characterization revealed that the growth direction was $\langle 001\rangle$. Rocking curves for various Bragg reflections in the $\{110 ;$ zone were recorded. The crystal was then mounted with its growth direction vertical to the goniometer table and the rocking curves for all the reflections possible in the $\{001 ;$ zone were recorded. This also 


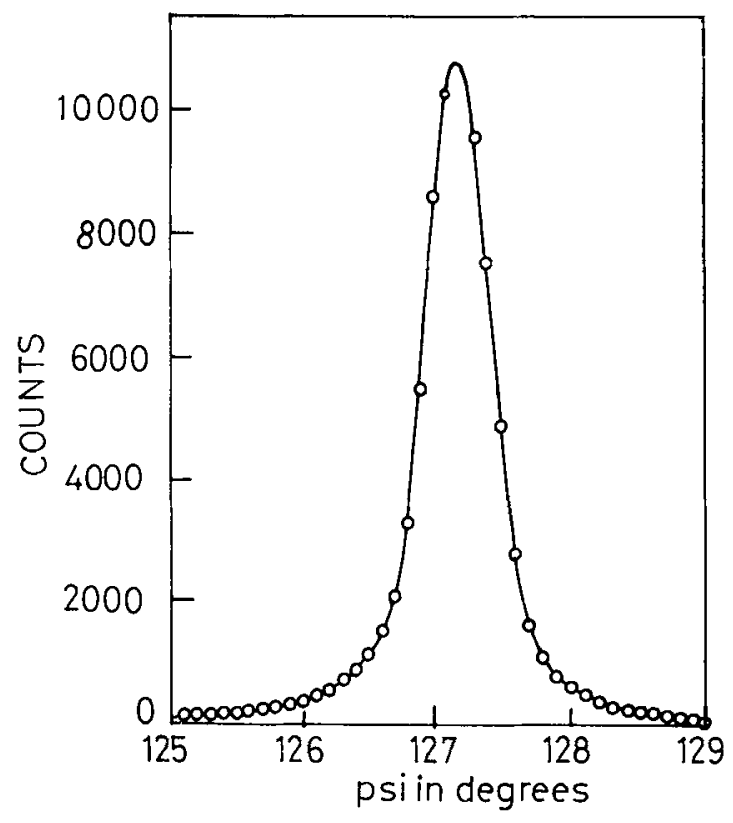

Figure 4. A typical rocking curve of (111) reflection from the copper crystal.

confirmed the growth direction to be $\langle 001\rangle$. To measure the reflectivity of the crystal, the detector was set in the incident monochromatic beam direction (i.e. zero position of scattering angle), and rocking curves of the transmitted beam with crystal in various Bragg positions were recorded.

\section{Results and discussion}

A typical rocking curve is shown in figure 4 . The solid line is just a guide to the eye. The shape of the rocking curves indicates the gross perfection of the crystal. The rocking curves (figure 5) are structure free and have symmetrical shape. This indicates good quality of the grown crystal. The full widths at half maxima, for the various reflections and corresponding values of the mosaic spreads calculated using (3), are given in table 2. These values of mosaic spread have been referred by Dorner (1971) as effective mosaic widths. (The mosaic spread of the monochromator crystal $\beta_{1}$ was determined to be 14 min.arc by plotting the rocking curve of a perfect germanium crystal in the parallel configuration with the monochromator). Figure 6 gives the plot of half widths versus dispersion parameter a of the reflections. It may be noted that width of the reflecting curve is minimum in the region $\theta=29^{\circ}$. The mosaic spread given by (4) was calculated using the $\mathrm{Cu}(002)$ reflection. The $(0002)$ of Be has a d-spacing of $1.8011 \AA$ which is close enough to $\mathrm{Cu}(002)$ spacing of $1.807 \AA$. Effects of resolution on the rocking curves are eliminated in this so called dispersion free geometry. The value of mosaic spread calculated in this way was found to be $\sim 14$ min.arc.

The rocking curves for the transmitted beam for various reflections showed that the reduction in intensity of the transmitted beam did not correspond exactly to the 


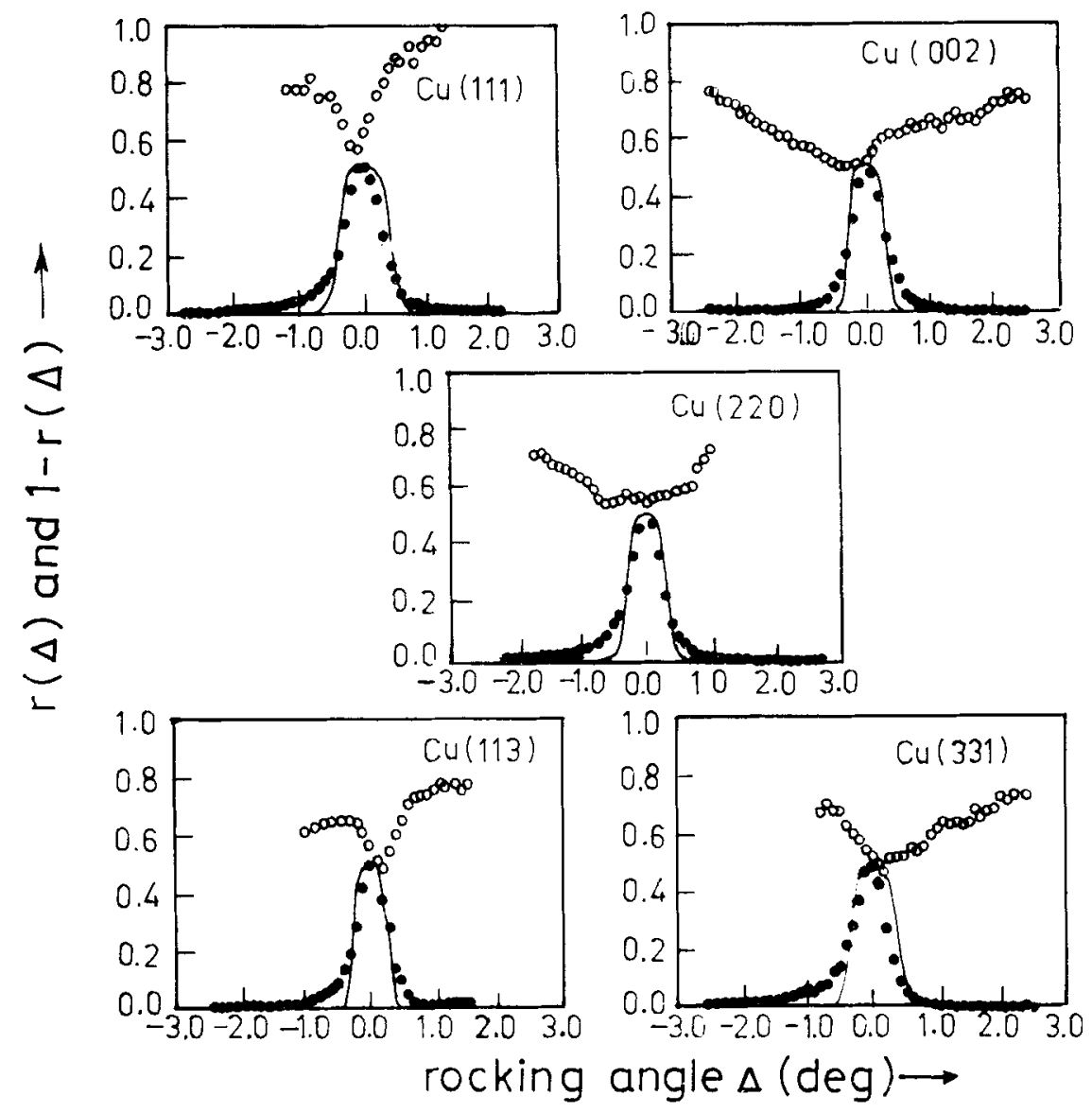

Figure 5. Rocking curves for the copper crystal: $(\mathcal{O})$ ) experimental points (diffracted); $(-\ldots$ calculated curve (diffracted); and $(\mathrm{O} \bigcirc \mathrm{O})$ experimental points (transmitted).

Table 2. Mosaic structure and reflectivity parameters of the copper crystal.

\begin{tabular}{lccccc}
\hline $\begin{array}{c}\theta \\
(h k l)\end{array}$ & $(\mathrm{deg})$ & $\mathrm{a}=\tan \theta / \tan \theta_{\mathrm{m}}$ & $\begin{array}{c}\text { FWHM } \\
(\operatorname{arc} . \min )\end{array}$ & $\begin{array}{c}\text { Mosaic spread } \\
(\operatorname{arc} . \min )\end{array}$ & $\begin{array}{c}\text { Reflectivity } \\
(\%)\end{array}$ \\
\hline$(111)$ & 14.56 & 0.8533 & 41.4 & 38.48 & 46 \\
$(002)$ & 16.87 & 0.9962 & 33.0 & 30.06 & 50 \\
$(220)$ & 24.24 & 1.4790 & 33.0 & 27.90 & 25 \\
$(113)$ & 28.77 & 1.8040 & 30.0 & 21.75 & 25 \\
$(331)$ & 39.24 & 2.6841 & 33.0 & 21.31 & 10 \\
\hline
\end{tabular}

diffracted intensity over the whole curve. This would be largely due to absorption of neutrons in the crystal.

The reflectivity of the crystal in various directions was calculated at this stage by taking ratio of the diffracted intensity to the incident intensity, which is the transmitted intensity away from the Bragg peak. These are quoted in table 2 . The diffracted and 


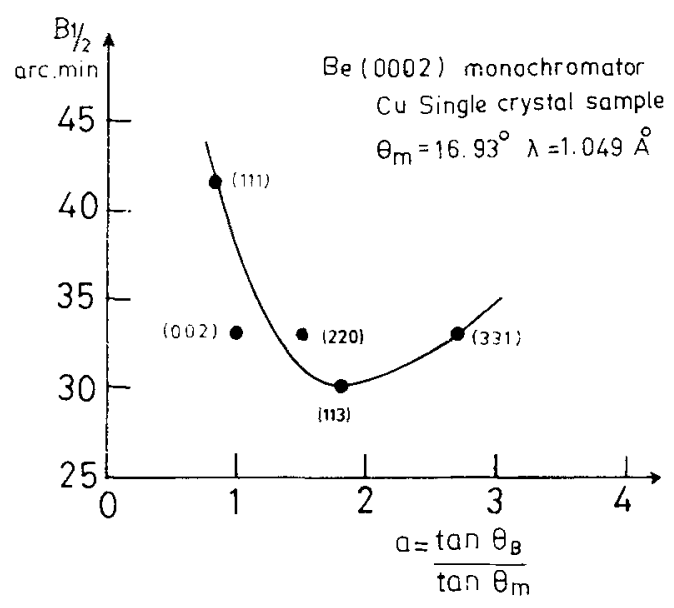

Figure 6. Plot of half-widihs of rocking curves vs dispersion parameter a for the different reflections.

transmitted intensities were then corrected for the absorption (Chidambaram 1980) and extinction (Darwin 1922) effects. The calculated reflectivity obtained using (3) was fitted to this corrected data by refining the true mosaic width given by (2). The results are shown in figure 5 . The calculated reflectivity curve shows a flat maximum at a value of 0.5 .

\section{Conclusions}

In summary, mosaic structure and reflectivity of a large size as grown copper crystal have been investigated using neutron diffraction. The crystal was found to be of good quality as indicated by the symmetric and structure free shapes of the rocking curves. The crystal has a mosaic spread of 14 min.arc and reflectivity of $\sim 50 \%$ which can be considered as very good values. The crystal, therefore, can be used for making monochromators after slicing along (002) plane.

\section{Acknowledgement}

The authors thank Dr K R Rao for his encouragement and interest in this work.

\section{References}

Arndt U W and Willis B T M 1966 Single crystal diffractometry (Cambridge University Press) p. 211

Bacon G E and Lowde R D 1948 Acta Crystallogr. 1303

Caglioti G and Ricci F P 1962 Nucl. Instrum. Meth. 15155

Chidambaram R 1980 in Proc: international school on crystallographic computing (eds) R Diamond, $S$ Ramaseshan and K Venkatesan (Bangalore: Indian Academy of Sciences) p. 2.01

Darwin C G 1922 Philos. Mag. 43800

Dorner B 1971 J. Appi Crist. 4185 